\title{
Zur inhaltlichen Schwerpunktsetzung im Rahmen der verbindlichen Übung „Digitale Grundbildung“ an österreichischen Mittelschulen
}

\author{
Stefan Oppl ${ }^{1}$, Wolfgang Fuchs ${ }^{2}$, Martin Dobiasch ${ }^{1}$
}

https://doi.org/10.53349/resource.2021.i16.a990

\section{Zusammenfassung}

Seit dem Schuljahr 2018/19 wird in Österreich die verbindliche Übung „Digitale Grundbildung“ flächendeckend in der Sekundarstufe I umgesetzt. Der Lehrplan ist durch eine sehr umfassend und detailliert ausgearbeitete Charakterisierung der zu adressierenden Themenfelder gekennzeichnet, in der die inhaltliche Heterogenität des Feldes sichtbar wird. In der Umsetzung herrscht weitgehende Flexibilität hinsichtlich des Ausmaßes, der Art der Verankerung und der inhaltlichen Schwerpunktsetzung. Vor diesem Hintergrund widmet sich die vorliegende Studie der Frage nach der konkreten Umsetzung der verbindlichen Übung in der Schulpraxis. Dazu wurden im Schuljahr 2019/20 Lehrpersonen und Schulleitungen aller Vorarlberger Mittelschulen befragt. Betrachtet wurde neben den Rahmenbedingungen der Umsetzung, den konkreten Umsetzungsformen und den Weiterbildungsbedarfen auch die Wichtigkeitswahrnehmungen der im Lehrplan definierten acht Teilbereiche und deren Priorisierung in der Unterrichtspraxis. Die Ergebnisse zeigen insgesamt eine breit über Fächergrenzen hinweg getragene und ambitionierte Umsetzung der verbindlichen Übung in der Praxis. Inhaltlich zeigt sich ein heterogenes Bild, in dem jene Teilbereiche, die eine informatisch-technische Perspektive abbilden, tendenziell von einem Großteil der Lehrpersonen und Schulleitungen als weniger wichtig wahrgenommen und in der Umsetzung eher nachrangig behandelt werden. Der Artikel versucht abschließend, mögliche Gründe für dieses Ungleichgewicht zu identifizieren, die in weiterführenden Studien umfassender zu untersuchen wären.

\section{Keywords:}

Digitale Grundbildung

Informatische Bildung

Umsetzungsstudie

\section{Einleitung}

Im Schuljahr 2018/2019 wurde in Österreich flächendeckend die verbindliche Übung „Digitale Grundbildung“ an allen Schulen der Sekundarstufe I eingeführt (Baberi et al., 2018; Reisinger, 2018). Die zu unterrichtenden Themen und relevanten Bereiche wurden in einem vom Bundesminister für Bildung, Wissenschaft und Forschung verordneten Lehrplan (BMBWF, 2018) definiert. Ebenso wurde das zeitliche Ausmaß festgelegt: Mit zwei bis vier Wochenstunden an verpflichtender Digitaler Grundbildung über den gesamten Zeitraum der Sekundarstufe I sollen die Lernenden digitale Kompetenzen erwerben, durch die sie in der Lage sein sollen, Herausforderungen des 21. Jahrhunderts zu meistern. In der operativen Umsetzung herrscht weitgehende Flexibilität und Autonomie - so können die Inhalte sowohl in eigens dafür eingerichteten Stunden als auch integrativ in bestehenden Fächern unterrichtet werden, auch die organisatorische wie inhaltliche Verteilung über die vier Schuljahre der Sekundarstufe I kann an jedem Schulstandort autonom festgelegt werden. Inhaltlich legt der Lehrplan einen weiten Rahmen fest, der in acht Themenbereichen die Vielfalt der zu vermittelten Inhalte definiert: "Gesellschaftliche Aspekte von Medienwandel und Digitalisierung", "Betriebssysteme und StandardAnwendungen “, "Informations-, Daten- und Medienkompetenz", „Mediengestaltung“, ,Digitale Kommunikation und Social Media“, „Sicherheit", „Technische Problemlösung" und „Computational Thinking“. Er definiert für

\footnotetext{
${ }^{1}$ Universität für Weiterbildung Krems, Dr.-Karl-Dorrek-Straße 30, 3500 Krems.

E-Mail: stefan.oppl@donau-uni.ac.at, martin.dobiasch@donau-uni.ac.at

2E-Mail: eeducation.wf@gmail.com
} 
jeden dieser Teilbereiche auch umfassend Lernergebnisse, so dass die Inhalte durch insgesamt 105 "Can-Do"Aussagen detailliert charakterisiert werden. Die konkrete inhaltliche Schwerpunktsetzung obliegt ebenso wie die methodische Ausgestaltung wiederum den Schulstandorten bzw. den einzelnen Lehrpersonen. Die sich aus der thematischen Vielfalt ergebenden Herausforderungen in der Umsetzung des Unterrichts sowie der Umgang mit dem notwendigen Aufbau von Lehrkompetenz und der Entwicklung von entsprechenden Unterrichtsmaterialen wurden bereits in der Pilotphase im Jahr 2017/18 als kritisch identifiziert (Swertz, 2018). Mit der verpflichtenden Umsetzung im Schuljahr 2018/19 sind diese Herausforderungen in der Breite des österreichischen Schulwesens angekommen. Die COVID19-Pandemie ab dem Schuljahr 2019/20 und der damit verbundene Entfall eines wesentlichen Teils des Präsenzunterrichts hat hier einerseits zu zusätzlichen Herausforderungen geführt, andererseits aber auch die Relevanz der Bildung entsprechender digitaler Kompetenzen verdeutlicht.

Vor dem Hintergrund der inhaltlichen Heterogenität der Themen, die im Rahmen der verbindlichen Übung „Digitale Grundbildung" adressiert werden sollen, hat dieser Beitrag das Ziel, deren operative Umsetzung aus Sicht der betroffenen Schulen und der involvierten Lehrpersonen und die dabei notwendigen Schwerpunktsetzungen näher zu untersuchen. Er betrachtet dazu die organisationalen Rahmenbedingungen, unter denen Schulen und Lehrpersonen die verbindliche Übung „Digitale Grundbildung“ implementieren, sowie die wahrgenommene Relevanz der inhaltlichen Schwerpunkte aus Sicht von Lehrpersonen und Schulleitungen. Durchgeführt wurde die Untersuchung in Form einer Befragung von Lehrpersonen und Schulleitungen der Mittelschulen in Vorarlberg unmittelbar vor Beginn der Auswirkungen der COVID19-Pandemie in Österreich. Dieser Artikel leistet somit einen Beitrag zu einem umfassenderen Verständnis der Umsetzung der verbindlichen Übung „Digitale Grundbildung“ im ersten Jahr nach ihrer flächendeckenden verpflichtenden Etablierung und trägt zur Verbesserung der bislang noch dürftigen Befundlage bei.

Im folgenden Abschnitt gehen wir auf die Hintergründe der Entwicklung der verbindlichen Übung „Digitale Grundbildung" im österreichischen Schulwesen ein und versuchen, die Genese der inhaltlichen Ausrichtung zu beleuchten. Abschnitt 3 beschreibt die Fragestellungen der Untersuchung sowie die angewendete Methodik. In Abschnitt 4 stellen wir entlang der Untersuchungsfragen die Ergebnisse der durchgeführten Studie dar, bevor wir diese in Abschnitt 5 vor dem Hintergrund der existierenden konzeptuellen Arbeiten im Feld diskutieren. Der Artikel schließt mit einer Identifikation der Handlungsfelder, die sich aus den Studienergebnissen ableiten lassen.

\section{Hintergrund - Digitale Grundbildung und deren Inhalte}

Die inhaltliche Heterogenität der verbindlichen Übung „Digitale Grundbildung“ spiegelt einen Diskurs wider, der in der Fachliteratur schon seit Jahren geführt wird. Die Frage danach, welche Lernergebnisse als grundlegende „digitale Kompetenzen“ für Schüler*innen anzustreben sind, ist geprägt von den fachlichen Perspektiven, die die jeweils handelnden Personen einnehmen. War der Diskurs seit Beginn der 2000er-Jahre geprägt von einer operativ wenig im Austausch stehenden Dualität aus einer medienbildnerischen und informatisch-technischen Perspektive (beschrieben etwa in (Brandhofer, 2014) oder (Tulodziecki, 2016)), wird schon ebenso lange der Anspruch, eine integrative Sicht auf digitale Kompetenzen einzunehmen (etwa in (Schelhowe, 2007), (Herzig, 2016) oder (Dengel, 2017), umfassend beschrieben auch in (Brandhofer \& Wiesner, 2018)).

Die operative Abbildung dieses Anspruchs auf konkrete Kompetenzmodelle für Lernende einerseits und Curricula andererseits stellt sich ob der Vielfalt der von unterschiedlichen Akteur*innen als relevant erachteten Themenfelder als nicht trivial dar. Einen breit rezipierten Ansatz zur Spezifikation möglicher Kompetenzen und deren Gliederung stellt beispielhaft herausgegriffen das DigComp-Framework der Europäischen Union dar (Ferarri, 2013), das seit der ursprünglichen Veröffentlichung mehrfach überarbeitet und ergänzt wurde und in fünf Kategorien strukturiert versucht, unterschiedliche Perspektiven abzubilden. Dies erfolgt großteils beispielgetrieben, eine konsistente konzeptuelle Basis ist hier jedoch nicht vorhanden (Brandhofer \& Wieser, 2018). Ein Modell, das sich explizit der Entwicklung einer disziplinenübergreifenden Sicht widmet, ist das „Dagstuhl-Dreieck“ (Gesellschaft für Informatik, 2016), in dem gleichberechtigt eine funktionsorientierte technologische (informatorische) Perspektive, eine nutzungsorientierte Anwendungsperspektive und eine wirkungsbezogene gesellschaftlich-kulturelle Perspektive auf Phänomene der "digitalen, vernetzten Welt" eingenommen wird. Dieses Modell wird seit seiner initialen Publikation ebenfalls vielfältig rezipiert und als Ausgangspunkt für die Ableitung konkreterer Kompetenzmodelle bzw. didaktischer Rahmenwerke verwendet (etwa bei Brandhofer et al., 2019). Die inhaltlichen Schwerpunkte der verbindlichen Übung „Digitale Grundbildung" referieren nicht explizit auf eines dieser Rahmenwerke, decken aber ähnliche Teilaspekte ab, wenngleich die Zusammenhänge sich nicht immer nachvollziehbar erschließen lassen (Brandhofer et al., 2019, S. 327). 
Auffällig ist diese Ambiguität etwa in der Betrachtung von "Computational Thinking“, das als einer der acht Teilbereiche der verbindlichen Übung „Digitale Grundbildung“ "angeführt ist. Im DigComp-Framework wird dieser Begriff genauso wenig verwendet wie im Dagstuhl-Dreieck. Betrachtet man die Lernergebnisse, die in der Verordnung der verbindlichen Übung dafür formuliert werden, so ist ein klarer Bezug zu informatischen Fragestellungen zu erkennen (wie etwa auch bei Dengel \& Heuer, 2018), insbesondere zur Formulierung von Algorithmen, bis hin zur Erstellung von Software. Dem hingegen wird in den letzten Jahren der Begriff "Computational Thinking" unter Bezugnahme auf Wing (2006) im Fachdiskurs umfassender verstanden (Brandhofer et al., 2019) und als "Schlüsselkompetenz" (Wiesner \& Schreiner, 2020) bezeichnet, die es ermögliche, „Herausforderungen und Probleme einer digitalen Welt [...] zu lösen und [...] die Lösungen in variablen, sowohl konkreten als auch komplexen Situationen erfolgreich, viabel und verantwortungsvoll unter einer anwendungsbezogenen, technologischen, wissenschaftlichen und einer gesellschaftlich-kulturellen Perspektive nutzen zu können." - In diesem Vorschlag steht "Computational Thinking" nicht neben anderen Aspekten digitaler Kompetenzen, sondern bildet gleichsam einen breiteren didaktischen Schirm über diese. Die offensichtliche Dichotomie des Begriffs "Computational Thinking“ wird auch von Steiner \& Himpsl-Gutermann (2020) hervorgehoben, die letztlich die handelnden Pädagog*innen in der Position und Verantwortung sehen, hier durch entsprechende didaktische Rahmenbedingungen diese Ambiguität situationsspezifisch aufzulösen und eine Verankerung in der Lebensrealität der Lernenden zu ermöglichen.

Insgesamt zeigt sich also ein Bild, in dem die potentiell relevanten Teilaspekte digitaler Grundbildung weitgehend kohärent identifiziert werden können und sich auch im Lehrplan der verbindlichen Übung "Digitale Grundbildung" wiederfinden (wenn auch mit unterschiedlichen Strukturierungen). Heterogen bleiben die Verankerung der informatischen Perspektive im Allgemeinen und die Rolle von "Computational Thinking“ im Speziellen - für Letztere existieren unterschiedlich umfassende Begriffsverständnisse, die ein konkretes Fassen der Inhalte erschweren. In diesem Sinne wird in der Untersuchung auch zu klären sein, welche Auswirkung diese Ambiguität auf die Umsetzung der entsprechenden Teilbereiche im Rahmen der verbindlichen Übung "Digitale Grundbildung" in der Schulpraxis hat.

\section{Methodik}

Ziel dieses Artikels ist es, die Implementierung der verbindlichen Übung „Digitale Grundbildung“ an Mittelschulen zu untersuchen und dabei insbesondere den Umgang mit dem Spannungsfeld zu betrachten, das sich aus der inhaltlichen Heterogenität der zu adressierenden Themen ergibt. Dazu sind die in die Implementierung der digitalen Grundbildung in der schulischen Praxis aktiv involvierten Personengruppen relevante Untersuchungsteilnehmer*innen. Diese umfassen zum einen die Gruppe der Schulleitungen, die an ihren jeweiligen Schulen für die Ausgestaltung der Rahmenbedingungen zuständig sind. Da ein erheblicher Teil der eingeführten Digitalen Grundbildung autonome Entscheidungen zulässt bzw. erfordert, fällt der Schulleitung hier ein maßgebliches gestalterisches Element zu. Zum anderen prägen die Einführung der Digitalen Grundbildung vor allem auch die Lehrpersonen mit, die diese neuen Elemente im Unterricht vermitteln sollen.

\subsection{Untersuchungsdesign}

Um die Untersuchungsschwerpunkte abbilden zu können, wurden drei Themenfelder identifiziert, zu denen Befragungsitems formuliert wurden. Diese Themenfelder lassen sich durch drei untersuchungsleitende Fragen (UF) charakterisieren:

- UF1: In welchem Ausmaß und in welcher Form werden Stunden der Digitalen Grundbildung an den Schulen angeboten?

- UF2: Welche Bedeutung wird den acht Bereichen der Digitalen Grundbildung seitens der Schulleitungen sowie der Lehrpersonen zugestanden und welche Einflussfaktoren können identifiziert werden?

- UF3: Wie wird Angebot und Bedarf zu Fort- und Weiterbildung von Schulleitungen und Lehrpersonen wahrgenommen?

Je nach inhaltlicher Schwerpunktsetzung der Themenfelder wurden jeweils die Schulleitungen, die Lehrpersonen oder beide Zielgruppen zu den einzelnen Untersuchungsfragen befragt. Zur Operationalisierung wurde ein Fragebogeninstrument entwickelt, das folgende Skalen enthielt (strukturiert nach Untersuchungsfragen, in Klammer ist angeführt, welche Skala in welchem Fragebogen enthalten war - SL: Schulleitung, LP: Lehrperson): 
- UF1:

0 Stundenausmaß integrativer Unterricht (SL) - 4 Ausprägungen: $1 \mathrm{~h}, 2 \mathrm{~h}, 3-4 \mathrm{~h}$, mehr als $4 \mathrm{~h}$

- Stundenausmaß im Stundenplan verankerter Unterricht (SL) - 4 Ausprägungen: $1 \mathrm{~h}, 2 \mathrm{~h}, 3-4 \mathrm{~h}$, mehr als $4 \mathrm{~h}$

- Bevorzugte Fächer für integrativen Unterricht (SL, LP) - Auswahl aus einer umfassenden Liste an Schulfächern, Mehrfachauswahl möglich

- Form der Umsetzung von Digitaler Grundbildung pro Schulstufe (LP): 4 Ausprägungen je Schulstufe 5-8: im Stundenplan verankerter Unterricht, integrativer Unterricht, beides, kein Unterricht

- Ausmaß der Umsetzung bei integrativem Unterricht pro Schulstufe (LP): 4 Ausprägungen je Schulstufe 5-8: keine - kurze Sequenzen - längere Abschnitte - überwiegende Teile des Unterrichts

- UF2:

- Wahrgenommene Wichtigkeit jedes der acht Bereiche der Digitalen Grundbildung (SL, LP): 4teilige Likert-Skala (sehr wichtig - unwichtig)

- Zuordnung jedes der acht Bereiche zu präferierten Schulstufen, in denen diese adressiert werden sollen (LP): 7 Ausprägungen je Bereich: 5., 6., 7., 8. Schulstufe, früher (Volksschule), später (weiterführende Schule), nicht notwendig

- Wahrgenommene drei wichtigste Teilbereiche der digitalen Grundbildung (LP): 3 aus 8 Bereichen zu wählen

- UF3:

○ Wahrgenommenes Ausmaß an Fortbildungsangeboten (SL, LP): 3 Ausprägungen: zu wenig, ausreichend, zu viel

- Ausmaß der Lehrpersonen im Kollegium, die eine Fortbildung im Bereich der digitalen Grundbildung in Anspruch genommen haben (SL): keine, bis zu $25 \%, 25-50 \%$, mehr als $50 \%$

- Wunsch nach mehr Fortbildungsangeboten in den unterschiedlichen Bereichen der digitalen Grundbildung (LP): aus 8 Bereichen zu wählen, Mehrfachauswahl möglich

- Umfeld- und mögliche Einflussfaktoren:

- Geschlecht (LP)

$\circ$ Geprüfte Fächer (LP)

- Dienstjahre (LP)

- Anzahl der Lehrpersonen an der Schule (SL)

- Mitgliedschaft bei eEducation Austria (SL)

- Anzahl der facheinschlägig geprüften Lehrpersonen an der Schule (SL)

- Anzahl der nicht facheinschlägig ausgebildeten Lehrpersonen, die an der Schule in der digitalen Grundbildung tätig sind (SL)

\subsection{Durchführung}

Die Befragung wurde in Kooperation mit der Stabsstelle Kommunikation und Schulpartnerschaft der Bildungsdirektion Vorarlberg durchgeführt. Der Start der Befragung fand im Rahmen einer landesweiten Schulleitungstagung Ende November 2019 statt. Dort wurden die Schulleitungen von der Bildungsdirektion gebeten, möglichst zahlreich an der Befragung teilzunehmen. Außerdem wurde ein Flyer an die anwesenden Schulleitungen verteilt, welcher einen QR-Code sowie einen Link zur Befragung der Lehrpersonen enthielt. Die Befragung endete am 20. Dezember 2019 mit Beginn der Weihnachtsferien.

Laut der Schulstatistik für Vorarlberg (Amt der Vorarlberger Landesregierung, 2019) gab es im Schuljahr 2017/18 in Vorarlberg 58 Mittelschulen mit dementsprechend ebenso vielen Schulleitungen und 1881 Lehrpersonen (588 männlich; 1293 weiblich). An der Befragung der Schulleitungen nahmen 24 Personen teil, was einer Beteiligung von rund 40 \% entspricht. Der Fragebogen für Lehrpersonen wurde von 64 Personen ausgefüllt. Aufgrund der mangelhaften Datenlage zu tatsächlich in der Digitalen Grundbildung tätigen Lehrpersonen ist hier eine prozentuelle Angabe der Beteiligung nur abschätzbar. Geht man von den Angaben der Schulleitungen aus, die an der Befragung teilgenommen haben, partizipieren an den Schulen, deren Leitungen an der Befragung teilgenommen haben, 141 der insgesamt tätigen 613 Lehrpersonen aktiv an der Umsetzung der Digitalen Grundbildung - dies entspricht einem Anteil von $23 \%$. Legt man diesen Anteil auf die Gesamtheit der in Vorarlberg tätigen Lehrpersonen um, so ist in der Gruppe der Lehrpersonen von einer Beteiligung von etwa $15 \%$ 
auszugehen (64 von kalkulatorisch 432 im Betrachtungszeitraum in der digitalen Grundbildung tätigen Lehrpersonen).

\section{Ergebnisse}

Dieser Abschnitt stellt die Ergebnisse der Befragung entlang der formulierten Untersuchungsfragen dar. Dabei wird zur Fokussierung des Artikels selektiv auf jene Aspekte eingegangen, die relevante Auffälligkeiten hinsichtlich der Fragestellungen zeigen. Der gesamte Datensatz ist auf Anforderung bei den Autoren zu beziehen.

\subsection{Teilnehmende Schulen und Lehrpersonen}

Alle an der Untersuchung teilnehmenden Lehrpersonen geben an, aktiv im Bereich der Digitalen Grundbildung zu unterrichten. Die Lehrerfahrung (gemessen am Dienstalter) und das Geschlecht der teilnehmenden Lehrpersonen sind in Tabelle 1 dargestellt.

\begin{tabular}{|l|c|c|c|c|}
\hline Dienstalter/Geschlecht & $m$ & $w$ & $d / k A$ & Gesamt \\
\hline 0-5 Jahre & 6 & 9 & 1 & 16 \\
\hline 6-10 Jahre & 3 & 9 & 0 & 12 \\
\hline 11-20 Jahre & 3 & 9 & 0 & 12 \\
\hline über 20 Jahre & 13 & 10 & 1 & 24 \\
\hline Gesamt & 25 & 37 & 2 & 64 \\
\hline
\end{tabular}

Tabelle 1: Verteilung nach Alter und Geschlecht der teilnehmenden Lehrpersonen

59 der 64 teilnehmenden Lehrpersonen machen Angaben über die geprüften Fächer. Die Verteilung der Angaben über geprüfte Fächer (Mehrfachangaben möglich) der teilnehmenden Lehrpersonen sind in Tabelle 2 dargestellt.

\begin{tabular}{|l|c|c|l|c|c|}
\hline Fach & $f$ & Anteil & Fach & $f$ & Anteil \\
\hline Mathematik & 31 & $52,5 \%$ & Sonstige & 7 & $11,9 \%$ \\
\hline Englisch & 12 & $20,3 \%$ & Chemie & 6 & $10,2 \%$ \\
\hline Geographie & 11 & $18,6 \%$ & Bildn. Erziehung & 5 & $8,5 \%$ \\
\hline Deutsch & 10 & $16,9 \%$ & Religion & 5 & $8,5 \%$ \\
\hline Sport & 10 & $16,9 \%$ & Musik & 4 & $6,8 \%$ \\
\hline Geschichte & 8 & $13,6 \%$ & Informatik & 4 & $6,8 \%$ \\
\hline Biologie & 7 & $11,9 \%$ & Geom. Zeichnen & 3 & $5,1 \%$ \\
\hline Physik & 7 & $11,9 \%$ & Italienisch & 2 & $3,4 \%$ \\
\hline
\end{tabular}

Tabelle 2: Verteilung der geprüften Fächer der teilnehmenden Lehrpersonen

Die teilnehmenden Lehrpersonen bezeichnen sich selbst als tendenziell sehr affin zu Themen der digitalen Bildung (4-teilige Likert-Skala, 1 ... sehr affin, 4 ... nicht affin; $n=64$, mean=1,64, std-dev=0,675, $\min =1, \max =3$ ).

Der Großteil der an der Umfrage beteiligten Schulen setzt im Bereich der Digitalen Grundbildung sowohl geprüfte Lehrpersonen (Selbsteinschätzung der Schulleitungen, z. B. Informatik oder einschlägige Weiterbildungskurse mit Bezug zu digitalen Inhalten) als auch ungeprüfte Lehrpersonen ein. An drei Schulen waren keine facheinschlägig geprüften Lehrpersonen tätig, an einer Schule unterrichten keine ungeprüften Lehrpersonen im Bereich der Digitalen Grundbildung. Insgesamt sind im arithmetischen Mittel 24,5\% der Lehrpersonen einer Schule im Bereich der Digitalen Grundbildung unterrichtend tätig ( $n=24$, std-dev=17,0 \%, $\min =10,7 \%, \max =100 \%)$. Davon ist im arithmetischen Mittel etwa $1 / 3$ facheinschlägig geprüft $(n=24$, mean=7,4 $\%$, std-dev=5,1 \%, $\min =0,0 \%, \max =17,7 \%$ ) und die übrigen Lehrpersonen sind nicht facheinschlägig ausgebildet 
( $n=24$, mean=17,1 \%, std-dev=18,4 \%, min=0,0\%, max=100\%). 15 der 24 teilnehmenden Schulen sind Mitglied der Initiative eEducation Austria des BMBWF.

\subsection{UF 1 - Unterrichtsform und Verankerung}

Hinsichtlich der Unterrichtsform der digitalen Grundbildung zeigt sich, dass in allen Schulen über die Schulstufen hinweg ein kombinierter Ansatz verfolgt wird, bei dem einzelne Aspekte im Rahmen explizit im Stundenplan verankerte Fächer unterrichtet werden, andere aber integrativ in anderen Fächern miteinbezogen werden. Tabelle 3 stellt die Verteilung der Angaben der Schulleitungen dar. Tabelle 4 zeigt, wie die beiden Unterrichtsformen kombiniert wurden. Eine gleichgewichtige Verteilung wurde angenommen, wenn beide Unterrichtsformen über die 4 Schuljahre hinweg im gleichen Ausmaß angeführt wurden. Eine stärkere Gewichtung von integrativem Unterricht wurde angenommen, wenn für die jeweilige Schule eine um eine Stufe auf der Skala höhere Ausprägung für integrative Unterrichtsformen gewählt wurde. Bei höherem Abstand auf der Skala wurde eine viel höhere Gewichtung von integrativem Unterricht angenommen. Analog sind die Ausprägungen „mehr explizit“ und „viel mehr explizit“ hinsichtlich einer stärkeren Gewichtung der explizit im Stundenplan verankerten Unterrichtsform zu verstehen.

\begin{tabular}{|l|c|c|c|c|}
\hline & \multicolumn{2}{|c|}{ explizit verankert } & \multicolumn{2}{c|}{ integrativ } \\
\hline & $f$ & Anteil & $f$ & Anteil \\
\hline nicht umgesetzt & 0 & $0,0 \%$ & 0 & $0,0 \%$ \\
\hline 1 Stunde & 3 & $12,5 \%$ & 7 & $29,2 \%$ \\
\hline 2 Stunden & 11 & $45,8 \%$ & 6 & $25,0 \%$ \\
\hline 3-4 Stunden & 7 & $29,2 \%$ & 7 & $29,2 \%$ \\
\hline mehr als 4 Stunden & 3 & $12,5 \%$ & 4 & $16,7 \%$ \\
\hline
\end{tabular}

Tabelle 3: Verteilung der Unterrichtsformen nach Angabe der Schulleitung $(n=24)$

\begin{tabular}{|l|c|c|}
\hline & $f$ & Anteil \\
\hline viel mehr integrativ & 4 & $16,7 \%$ \\
\hline mehr integrativ & 5 & $20,8 \%$ \\
\hline gleichgewichtig & 9 & $37,5 \%$ \\
\hline mehr explizit & 2 & $8,3 \%$ \\
\hline viel mehr explizit & 4 & $16,7 \%$ \\
\hline
\end{tabular}

Tabelle 4: Gewichtung der Unterrichtsformen nach Angabe der Schulleitung ( $n=24)$

Neben den Schulleitungen wurden auch die Lehrpersonen zu der Unterrichtsform befragt, in der sie die verbindliche Übung „Digitale Grundbildung“ je nach Schulstufe umsetzen. Die Antworten sind in Tabelle 5 zusammengefasst. Auffällig ist hier die Verschiebung hin zu integrativem Unterricht in der 7. und 8. Schulstufe zu Ungunsten der explizit im Stundenplan verankerten Unterrichtsform.

\begin{tabular}{|l|l|l|l|l|}
\hline & 5. SSt. & 6. SSt. & 7. SSt. & 8. SSt. \\
\hline integrativ & 16 & 15 & 23 & 25 \\
\hline explizit verankert & 19 & 17 & 8 & 9 \\
\hline beide Varianten & 11 & 16 & 10 & 9 \\
\hline nur punktuell & 8 & 4 & 5 & 4 \\
\hline keine Angabe & 10 & 12 & 18 & 17 \\
\hline
\end{tabular}

Tabelle 5: Unterrichtsform nach Schulstufen nach Angabe der Lehrpersonen $(n=64)$ 
Im Falle von integrativen Unterrichtsformen liegt es in der Kontrolle der Lehrpersonen, in welchem Ausmaß Aspekte der digitalen Grundbildung neben den eigentlichen Fachinhalten im Unterricht verankert werden. Wie in Abbildung 1 ersichtlich, zeigt sich hier eine Differenzierung nach Schulstufen. Nach Angabe der befragten Lehrpersonen fließen in den beiden niedrigeren Schulstufen diese Aspekte eher in kurzen Sequenzen neben den eigentlichen Fachinhalten in den Unterricht ein. In den höheren beiden Schulstufen werden überwiegend längere Unterrichtssequenzen der Vermittlung von Aspekten der digitalen Grundbildung gewidmet.
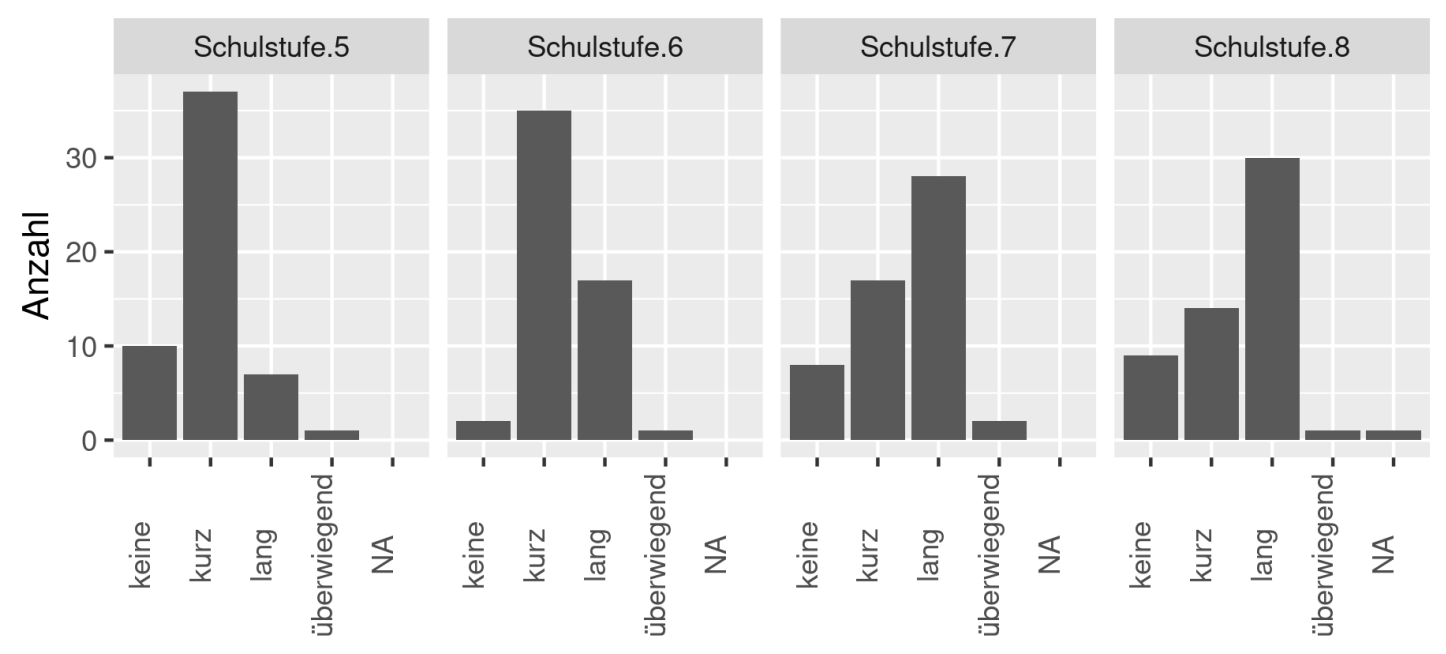

Abbildung 1: Angaben der Lehrpersonen über Umfang der Digitalen Grundbildung als integrativer Unterricht über Schulstufen hinweg

Hinsichtlich der von Lehrpersonen bevorzugten Fächer für integrativen Unterricht über Aspekte der digitalen Grundbildung zeigt sich die in Tabelle 6 dargestellte Verteilung (59 der 64 teilnehmenden Lehrpersonen machten hier Angaben, Mehrfachantworten waren möglich).

\begin{tabular}{|l|c|c|}
\hline Fach & $f$ & Anteil \\
\hline Mathematik & 28 & $47,5 \%$ \\
\hline Englisch & 13 & $22,0 \%$ \\
\hline Geographie & 13 & $22,0 \%$ \\
\hline Deutsch & 15 & $25,4 \%$ \\
\hline Geschichte & 11 & $18,6 \%$ \\
\hline Biologie & 12 & $20,3 \%$ \\
\hline Physik/Chemie & 13 & $22,0 \%$ \\
\hline Sonstige & 10 & $16,9 \%$ \\
\hline
\end{tabular}

Tabelle 6: Verteilung der Fächerpräferenzen für integrativen Unterricht $(n=59)$

Unter "Sonstige" sind in Tabelle 6 Fächer wie "Musik“, „Bildnerische Erziehung", "Religion“ (jeweils $\mathrm{f}=2$ ) und weitere einzeln genannte, aufgrund der Verwendung von Kürzeln nicht näher identifizierbare Fächer zusammengefasst.

\subsection{UF 2 - Rolle der acht Teilbereiche}

Die im Lehrplan angeführten acht Teilbereiche, die im Rahmen der digitalen Grundbildung adressiert werden sollen, wurden bereits in der Einführung skizziert. In der Befragung wurden sowohl Schulleitungen als auch Lehrpersonen hinsichtlich der wahrgenommenen Wichtigkeit abgefragt. Lehrpersonen wurden zusätzlich nach dem Zeitpunkt der Adressierung des jeweiligen Teilbereichs im Unterricht (nach Schulstufen) befragt. Zur kompakteren Darstellung in den nun folgenden Abbildungen und Tabellen werden folgende Kürzel zur 
Referenzierung der Teilbereiche verwendet: Ges ... Gesellschaftliche Aspekte von Medienwandel und Digitalisierung, BS ... Betriebssysteme und Standard-Anwendungen, IDM ... Informations-, Daten- und Medienkompetenz, M ... Mediengestaltung, KSM ... Digitale Kommunikation und Social Media, S ... Sicherheit, P ... Technische Problemlösung, CT ... Computational Thinking.

In der Befragung nach der Wichtigkeit der Teilbereiche auf einer 4-teiligen Likert-Skala (1 ... sehr wichtig, 4... sehr unwichtig) zeigt sich zwischen Schulleitungen und Lehrpersonen ein ähnliches Bild, wenngleich die Aussagen der Lehrpersonen stärker differenzieren. Abbildung 2 zeigt die Histogramme der Schulleitungen, Abbildung 3 jene der Lehrpersonen. Tabelle 7 gibt zusätzlich noch Mittelwert und Std-Abw. je Teilbereich und befragter Gruppe an ( $\left.n_{\text {schulleitung }}=24, n_{\text {Lehrperson }}=64\right)$.
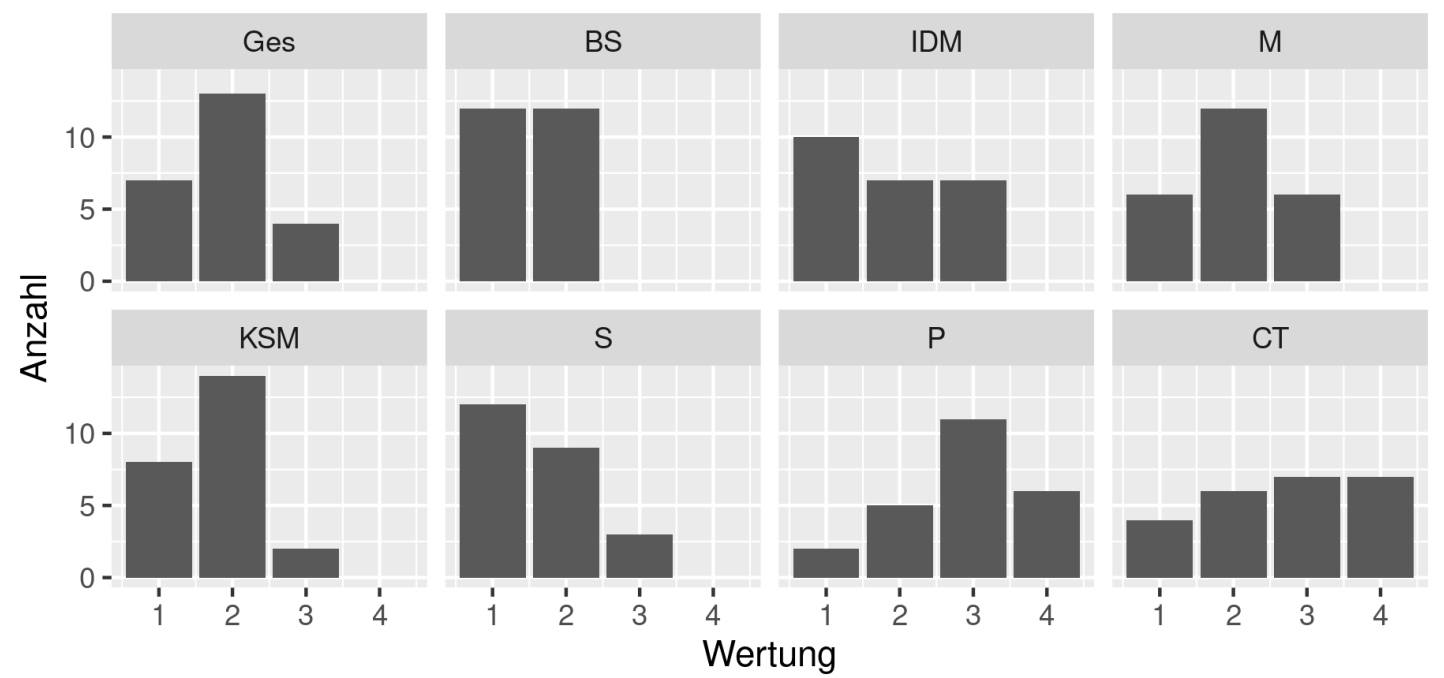

Abbildung 2: Wahrgenommene Wichtigkeit der Teilbereiche durch Schulleitungen (1 ... sehr wichtig, 4 ... sehr unwichtig).
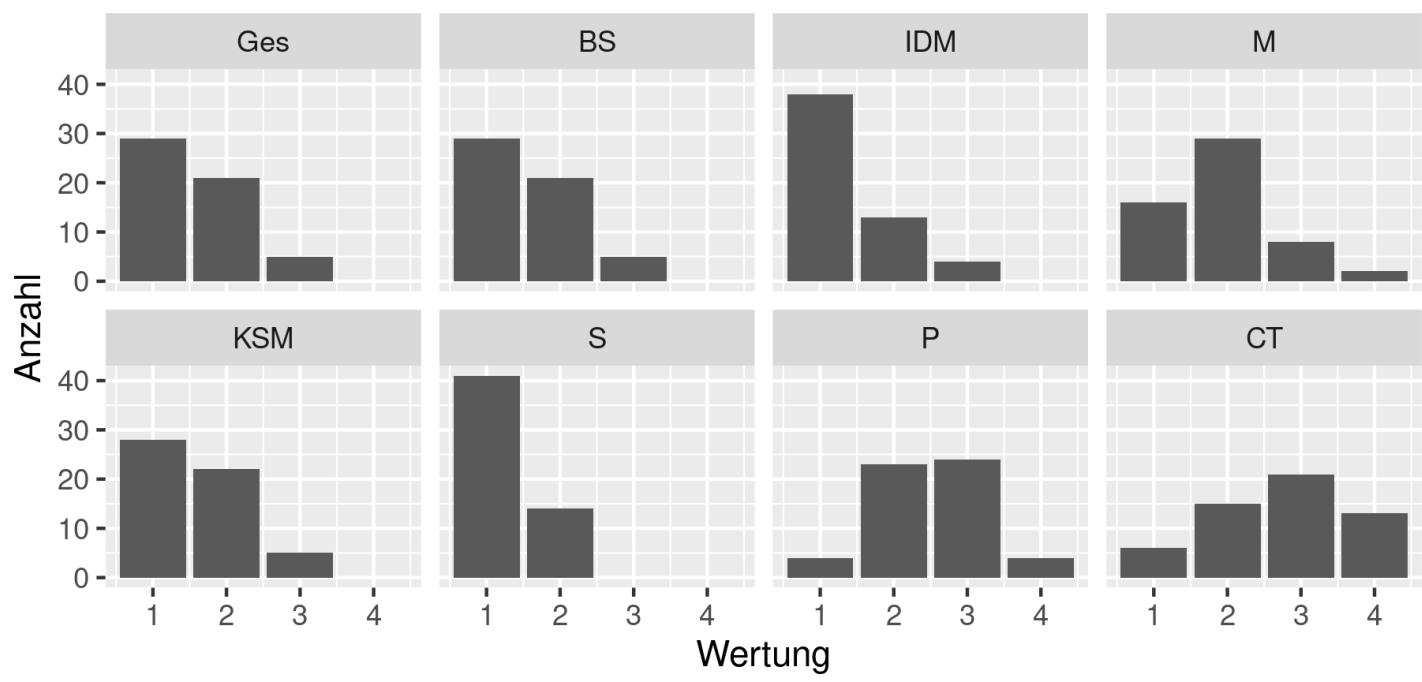

Abbildung 3: Wahrgenommene Wichtigkeit der Teilbereiche durch Lehrpersonen (1 ... sehr wichtig, 4 ... sehr unwichtig).

Von den Schulleitungen wurden die Teilbereiche „Betriebssysteme und Standard-Anwendungen“ und „Sicherheit“ im Mittel zwischen „sehr wichtig“ und „wichtig“ (Median 1.5) eingestuft, die Bereiche "Gesellschaftliche Aspekte von Medienwandel und Digitalisierung", „Informations-, Daten- und Medienkompetenz“, „Mediengestaltung“ und „Digitale Kommunikation und Social Media“ mit „wichtig“" (Median 2), während sich für „Technische Problemlösung “ und „Computational Thinking“ eine eher geringe Wertung zeigt („unwichtig“, Median = 3) (siehe Abbildung 2). Der Unterschied zu den Lehrpersonen ist gering, es werden aber tendenziell höhere Wertigkeiten wahrgenommen. Von den Lehrpersonen wurden die Teilbereiche "Gesellschaftliche Aspekte von Medienwandel und Digitalisierung“, „Betriebssysteme und StandardAnwendungen“, „Informations-, Daten- und Medienkompetenz", „Digitale Kommunikation und Social Media“ 
und „Sicherheit“ im Mittel (Median = 1) als „sehr wichtig“ eingestuft, „Mediengestaltung“ als „wichtig“, während sich für "Technische Problemlösung" und "Computational Thinking“ eine eher geringe Wertung zeigt („,unwichtig“, Median = 3) (siehe Abbildung 3).

\begin{tabular}{|l|c|c|c|c|}
\hline Teilbereich & \multicolumn{2}{|c|}{ Schulleitungen } & \multicolumn{2}{c|}{ Lehrpersonen } \\
\hline & mean & std-dev & mean & std-dev \\
\hline Ges & 1,88 & 0,68 & 1,58 & 0,71 \\
\hline IDM & 1,50 & 0,51 & 1,69 & 0,77 \\
\hline BS & 1,88 & 0,85 & 1,41 & 0,61 \\
\hline$M$ & 2,00 & 0,72 & 1,97 & 0,76 \\
\hline KSM & 1,75 & 0,61 & 1,62 & 0,71 \\
\hline$S$ & 1,63 & 0,71 & 1,27 & 0,45 \\
\hline$P$ & 2,88 & 0,90 & 2,48 & 0,76 \\
\hline$C T$ & 2,71 & 1,08 & 2,73 & 0,92 \\
\hline
\end{tabular}

Tabelle 7: Wahrgenommene Wichtigkeit der Teilbereiche (1 ... sehr wichtig, 4 ... sehr unwichtig)

Zusätzlich zur absoluten Wichtigkeit wurden Lehrpersonen separat nach den drei in ihrer Wahrnehmung wichtigsten Teilbereichen befragt. Tabelle 8 zeigt die Verteilung der Antworten hierbei (64 Personen machten hier Angaben, es waren bis zu drei Nennungen ohne weitere Reihung möglich, eine Person wählte nur zwei Teilbereiche aus).

\begin{tabular}{|l|c|c|}
\hline Teilbereich & $f$ & Anteil \\
\hline Ges & 23 & $35,9 \%$ \\
\hline IDM & 31 & $48,4 \%$ \\
\hline$B S$ & 32 & $50,0 \%$ \\
\hline$M$ & 16 & $25,0 \%$ \\
\hline KSM & 31 & $48,4 \%$ \\
\hline$S$ & 43 & $67,2 \%$ \\
\hline$P$ & 5 & $7,8 \%$ \\
\hline$C T$ & 10 & $15,6 \%$ \\
\hline
\end{tabular}

Tabelle 8: Verteilung der Nennungen der drei wichtigsten Teilbereiche $(n=64)$

Auch hier zeigt sich ein ähnliches Bild wie in der obigen Auswertung, wobei die Unterscheidung der Präferenzen ob der geforderten Auswahl und Fokussierung auf drei Bereiche hier klarer zu Tage tritt. Deutlich zeigt sich auch hier die niedrige Priorisierung der technisch-informatisch orientierten Teilbereiche „Technische Problemlösung“ und "Computational Thinking", während der Teilbereich "Sicherheit" von mehr als zwei Drittel aller Lehrpersonen angeführt wird.

Die wahrgenommene Wichtigkeit des Teilbereichs "Sicherheit" zeigt sich auch in der Darstellung der Präferenzen für Unterricht in den unterschiedlichen Teilbereichen nach Schulstufen. Lehrpersonen hatten hier für jeden Teilbereich die Möglichkeit, anzugeben, in welcher/n Schulstufe(n) diese adressiert werden sollten (Mehrfachauswahl möglich). Zudem konnte angeführt werden, wenn ein Teilbereich vor der 5. oder nach der 8. Schulstufe adressiert werden sollte oder wenn er als nicht relevant erachtet wurde. Tabelle 9 zeigt die Ergebnisse dieser Einordnung in absoluten Zahlen (n=64). Abbildung 4 visualisiert die gleichen Ergebnisse in über die Schulstufen aggregierter Form. Auffällig ist hier, dass die technischeren Teilbereiche „Technische Problemlösung" und "Computational Thinking“ erst in oder nach Ende der 8. Schulstufe breit als relevant eingeordnet werden, während die anderen Teilbereiche - auf unterschiedlichen Niveaus - relativ konstant über die Schuljahre verteilt präferiert unterrichtet werden. 
R\&E-SOURCE https://journal.ph-noe.ac.at Online Journal for Research and Education

\begin{tabular}{|l|c|c|c|c|c|c|c|}
\hline Teilbereich & früher & 5. SSt. & 6. SSt. & 7. SSt. & 8. SSt. & später & nicht notw. \\
\hline Ges & 2 & 11 & 18 & 28 & 19 & 5 & 2 \\
\hline$I D M$ & 2 & 24 & 31 & 24 & 13 & 3 & 0 \\
\hline$B S$ & 5 & 30 & 30 & 18 & 16 & 5 & 0 \\
\hline$M$ & 5 & 19 & 22 & 29 & 22 & 3 & 2 \\
\hline$K S M$ & 6 & 22 & 26 & 21 & 18 & 4 & 0 \\
\hline$S$ & 10 & 27 & 29 & 23 & 19 & 3 & 1 \\
\hline$P$ & 0 & 4 & 5 & 19 & 40 & 17 & 1 \\
\hline$C T$ & 4 & 12 & 9 & 14 & 29 & 25 & 7 \\
\hline
\end{tabular}

Tabelle 9: Präferenz für Unterricht zum jeweiligen Teilbereich nach Schulstufen

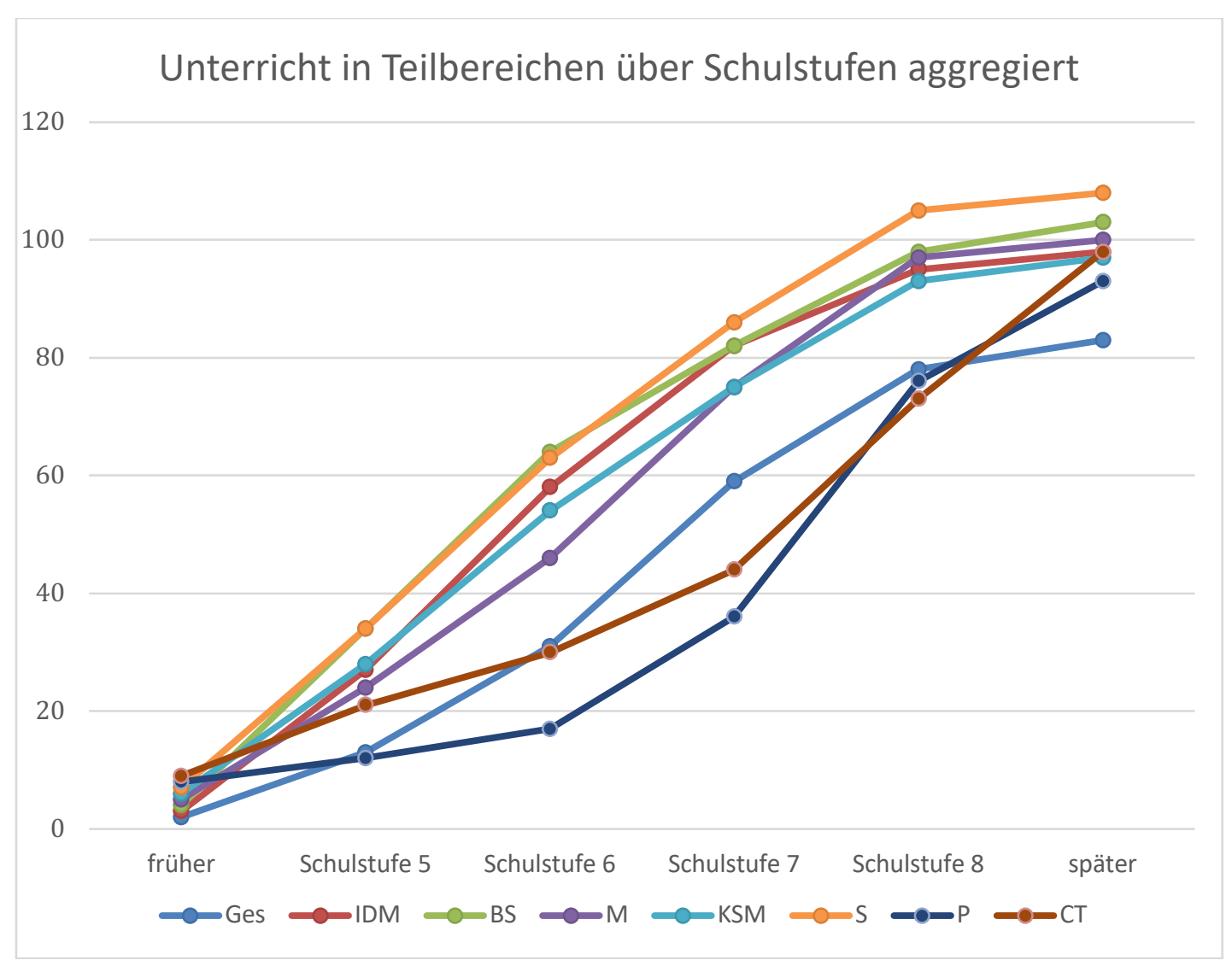

Abbildung 4: Präferenz für Unterricht in Teilbereichen über Schulstufen aggregiert (absolute Anzahl der Nennungen)

Weiters wurden mögliche Einflussfaktoren auf Präferenzen für einzelne Teilbereiche durch Lehrpersonen geprüft. Weder das Geschlecht der Lehrpersonen noch ihr Dienstalter noch die von den befragten Lehrpersonen als unterrichtet angegebenen Fächer zeigten einen Einfluss auf die Wichtigkeit der Teilbereiche - durchgeführte exakte Tests nach Fischer waren nicht signifikant. Insbesondere stehen Präferenzen für einzelne Teilbereiche nicht in signifikantem Zusammenhang mit den fachlichen Hintergründen der Lehrpersonen.

Da die abgefragten Einflussfaktoren keine Zusammenhänge mit der wahrgenommenen Wichtigkeit einzelner Teilbereiche zeigten, wurde zusätzlich eine Clusteranalyse durchgeführt, um etwaige gemeinsam auftretende Muster hinsichtlich gemeinsam als wichtig oder weniger wichtig eingestufter Teilbereiche identifizieren zu können.

Mittels k-Means Clustering wurden die Lehrpersonen anhand ihrer Antworten zur wahrgenommenen Wichtigkeit der Teilbereiche in drei Cluster eingeteilt ( $n=11 / 30$ / 14, siehe Tabelle 10). Eine zuvor durchgeführte optische Analyse von xy-Plots über die Summe der Fehler und die Anzahl möglicher Cluster zeigte, dass drei 
Cluster in den Datenmengen existieren können. Die ermittelten Cluster zeigten signifikante Unterschiede hinsichtlich der Einstufung von "Gesellschaftliche Aspekte von Medienwandel und Digitalisierung“ $(p<0,001)$, "Mediengestaltung" ( $p<0,01)$, Digitale Kommunikation und Social Media“ $(p<0,01)$, "Technische Problemlösung“ ( $p<0,001)$, „Computational Thinking“ ( $<0,001)$. Für „Betriebssysteme und StandardAnwendungen“, „Informations-, Daten- und Medienkompetenz", „Sicherheit“ wurde kein signifikanter Unterschied gefunden ( $p>0,05)$. Die jeweilig im Median angegebenen Wertigkeiten der Cluster sind in Tabelle 10 ersichtlich. Erneut war es in post-hoc Analysen nicht möglich, anhand der erhobenen Merkmale Lehrpersonen einem Cluster zuzuordnen. Es wurden hier verschiedenste Merkmalskombinationen untersucht. Eine genauere Betrachtung und inhaltliche Interpretation der Cluster und deren ermittelte Wertigkeiten legt nahe, dass der kleinste Cluster 1 Personen beinhaltet, die „Anwendungsorientierung” als Priorität haben („Betriebssysteme und Standard-Anwendungen“, „Mediengestaltung“, „Digitale Kommunikation und Social Media“, "Sicherheit“), während der größte Cluster 2 Gesellschaftliche Aspekte und Medienkompetenz („Gesellschaftliche Aspekte von Medienwandel und Digitalisierung“, „Informations-, Daten- und Medienkompetenz") als wichtig erachtet. Interessant ist an dieser Stelle aber auch Cluster 3, der sich durch eine hohe Wichtigkeitswahrnehmung aller Teilbereiche auszeichnet. Zusätzlich dazu fällt auf, dass es keinen Cluster gibt, in welchem die technischinformatischen Teilbereiche („Technische Problemlösung“, „Computational Thinking“) als prioritär betrachtet werden.

\begin{tabular}{|c|c|c|c|c|c|c|c|c|c|}
\hline Cluster & $n$ & Ges & $B S$ & $I D M$ & $M$ & $K S M$ & $S$ & $P$ & $C T$ \\
\hline 1 & 11 & 2 & 1 & 2 & 1 & 1 & 1 & 2 & 2 \\
\hline 2 & 30 & 1 & 2 & 1 & 2 & 2 & 1 & 3 & 3 \\
\hline 3 & 14 & 1 & 1 & 1 & 2 & 1 & 1 & 2 & 2 \\
\hline
\end{tabular}

Tabelle 10: Clustering Lehrpersonen nach wahrgenommener Wichtigkeit der Teilbereiche

Ein ähnliches, wenn auch weniger differenziertes Bild ergibt sich bei Anwendung des gleichen Prozedere auf die Angaben der Schulleitungen: auch hier ergeben sich drei Cluster $(n=12 / 7 / 5$, siehe Tabelle 11), die auf niedrigem Niveau, was die wahrgenommene Wichtigkeit der Teilbereich betriff, angesiedelt sind. Cluster 3 entspricht hier im Wesentlichen dem dritten Cluster der Lehrpersonen. Der kleinste Cluster 2 hebt auch hier die anwendungsnahen Teilbereiche Betriebssysteme und Standard-Anwendungen sowie Sicherheit in der wahrgenommenen Wichtigkeit hervor. Der größte Cluster 1 ist inhaltlich wenig differenziert, hervorzuheben ist hier einerseits die niedrige Wertung der technisch-informatischen Teilbereiche („Technische Problemlösung“, „Computational Thinking“) sowie das generell eher niedrigere Niveau der wahrgenommenen Wichtigkeit aller Teilbereiche im Vergleich zu den anderen Clustern der Schulleitungen und jenen der Lehrpersonen.

\begin{tabular}{|c|c|c|c|c|c|c|c|c|c|}
\hline Cluster & $n$ & Ges & BS & $I D M$ & $M$ & $K S M$ & $S$ & $P$ & $C T$ \\
\hline 1 & 12 & 2 & 2 & 2,5 & 2 & 2 & 2 & 3,5 & 4 \\
\hline 2 & 7 & 2 & 1 & 2 & 2 & 2 & 1 & 3 & 2 \\
\hline 3 & 5 & 1 & 1 & 1 & 1 & 1 & 1 & 2 & 1 \\
\hline
\end{tabular}

Tabelle 11: Clustering Schulleitungen nach wahrgenommener Wichtigkeit der Teilbereiche

Aus der explorativen Analyse der initialen Clusterbildung zeigte sich, dass sich jeweils zwei der drei Cluster (jeweils abgesehen von jenem, in dem die Mitglieder tendenziell alle Teilbereiche als wichtig eingeordnet haben) in der Einschätzung von thematisch verwandten Teilbereichen ähneln. Aus diesem Grund wurden die Teilbereiche im Rahmen einer ergänzenden Clusteranalyse entlang der im Lehrplan formulierten Lernergebnisse entsprechend der von Dengel (2017) auf Basis des Modells der Gesellschaft für Informatik (2016) vorgeschlagenen Perspektiven des „erweiterten Dagstuhl-Dreiecks“ gruppiert, um so etwaige Unterschiede zwischen den Clustern deutlicher sichtbar zu machen. Die Teilbereiche "Gesellschaftliche Aspekte von Medienwandel und Digitalisierung“, „Informations-, Daten- und Medienkompetenz" und „Digitale Kommunikation und Social Media“ wurden zu einem Bereich „Mediennutzung" (in (Dengel, 2017) „gesellschaftlich-kulturelle Perspektive“) zusammengefasst. Der Bereich „Computeranwendung“ (in (Dengel, 2017) „anwendungs- und wissensbezogene Perspektive“) umfasst die Teilbereiche „Betriebssysteme und Standard-Anwendungen“, „Mediengestaltung“ und „Sicherheit“. Die Teilbereiche „Technische Problemlösung“ und „Computational Thinking“ wurden zu einem Bereich „Technik/Informatik“ (in (Dengel, 2017) „informatische Perspektive") gruppiert. 
Für jeden der Bereiche wurde der Mittelwert der Wichtigkeitseinschätzung der jeweils inkludierten Teilbereiche gebildet. Für den Bereich „Mediennutzung“ gaben die Lehrpersonen im Mittel eine Wichtigkeit von 1,51 (std-dev = 0,42) an, während die Schulleitungen hier 1,83 (std-dev = 0,54) angaben. „Computeranwendung“ wurde mit 1,58 (std-dev = 0,38) bzw. 1,71 (std-dev = 0,43) und „Technik/Informatik“ mit 2,63 (std-dev = 0,70) und 2,79 (std-dev $=0,90$ ) bewertet. Es zeigt sich also auch hier, dass die Schulleitungen generell tendenziell niedrigere Wichtigkeitseinschätzungen als die Lehrpersonen vergeben. Wird nun analog zum oben beschriebenen Vorgehen ein k-means-Clustering durchgeführt, ergeben sich die jeweils drei in Tabelle 12 für Lehrpersonen und Tabelle 13 für Schulleitungen dargestellten Cluster.

\begin{tabular}{|c|c|c|c|c|}
\hline Cluster & $n$ & Mediennutzung & $\begin{array}{c}\text { Computer- } \\
\text { anwendung }\end{array}$ & $\begin{array}{c}\text { Technik/ } \\
\text { Informatik }\end{array}$ \\
\hline 1 & 22 & 1,333 & 1,333 & 3 \\
\hline 2 & 16 & 1,833 & 2 & 3,25 \\
\hline 3 & 17 & 1,333 & 1,333 & 2 \\
\hline
\end{tabular}

Tabelle 12: Clustering Lehrpersonen nach wahrgenommener Wichtigkeit der gruppierten Bereiche

\begin{tabular}{|c|c|c|c|c|}
\hline Cluster & $n$ & Mediennutzung & $\begin{array}{c}\text { Computer- } \\
\text { anwendung }\end{array}$ & $\begin{array}{c}\text { Technik/ } \\
\text { Informatik }\end{array}$ \\
\hline 1 & 7 & 2 & 2 & 4 \\
\hline 2 & 7 & 1,333 & 1,333 & 1,5 \\
\hline 3 & 10 & 2 & 1,833 & 3 \\
\hline
\end{tabular}

Tabelle 13: Clustering Schulleitungen nach wahrgenommener Wichtigkeit der gruppierten Bereiche

Es zeigt sich insgesamt ein etwas anderes, stärker differierendes Bild als im Rahmen der Einzelbetrachtung der Teilbereiche. Für die Lehrpersonen fällt nun auf, dass sich der größte Cluster 1 ( $n=22)$ und der zweitgrößte Cluster 3 ( $n=17)$ durch eine sehr hohe Wichtigkeitswahrnehmung des Bereichs "Mediennutzung" und „Computeranwendung“ auszeichnen und sich in der wahrgenommenen Wichtigkeit des Bereichs „Technik / Informatik“ unterscheiden. Während dieser in beiden Clustern hinter den anderen beiden Bereichen zurückliegt, wird er im Cluster 1 im Mittel als „unwichtig“ eingestuft, während er im Cluster 3 als „wichtig“ betrachtet wird. Cluster 2 zeigt generell niedrigere Wichtigkeitswahrnehmungen, kennzeichnend ist aber auch hier, dass „Technik / Informatik“ der einzige Bereich ist, der im Mittel als „unwichtig“ betrachtet wird.

Ein ähnliches Bild hinsichtlich der Cluster 1 und 3 zeigt sich für die Schulleitungen, wenngleich auch hier wieder auf niedrigerem wahrgenommenen Wichtigkeitsniveau. Der größte Cluster $3(n=10)$ lässt eine leichte Präferenz für den Bereich „Computeranwendung“ erkennen, „Technik / Informatik“ wird in beiden Clustern als „(sehr) unwichtig“ betrachtet. Cluster 2 ist hier anders ausgeprägt als bei den Lehrpersonen und zeichnet sich durch eine gleichmäßig hohe Wichtigkeitswahrnehmung aller drei Bereiche aus, wobei „Technik / Informatik“ hier leicht hinter den anderen beiden Bereichen liegt.

\subsection{UF 3 - Weiterbildungsbedarf}

Neben den bereits diskutierten unterschiedlichen Wahrnehmungen zur Wichtigkeit und Priorisierung der acht Teilbereiche wurden die Lehrpersonen auch zu deren Weiterbildungsbedarf befragt. Neben der Erhebung der Inanspruchnahme existierender Angebote wurde auch die wahrgenommene Notwendigkeit zusätzlicher Weiterbildungsangebote für jeden der acht Teilbereiche erhoben.

Befragt nach der Form der Weiterbildung, die im Bereich der digitalen Grundbildung bislang in Anspruch genommen wurde, geben $60,9 \%$ ( $f=39$ ) der teilnehmenden Lehrpersonen an, sich bislang autodidaktisch in Selbstlernphasen weitergebildet zu haben. Für 23,4 \% $(f=15)$ war dies die einzige Form der bisherigen Weiterbildung in diesem Bereich. 29,7\% ( $f=19$ ) geben an, bislang keine Weiterbildung zur digitalen Grundbildung in Anspruch genommen und sich auch nicht autodidaktisch weitergebildet zu haben. 32,8 \% ( $f=$ 21) der Teilnehmer*innen geben an, bislang zumindest einen Kurs im Ausmaß von maximal einem Tag in Anspruch genommen zu haben, $17,2 \%(f=11)$ geben an, ein höheres Ausmaß an Weiterbildungen in Anspruch genommen zu haben.

Das Angebot von Weiterbildungen im Bereich der Digitalen Grundbildung wird von 48,8\% $(f=28)$ der Lehrpersonen und von 33,3 \% ( $f=8)$ der Schulleitungen für ausreichend erachtet, 53,1 \% $(f=34)$ bzw. 58,3 \% ( $f$ 
= 14) sind der Meinung, dass das Ausmaß an angebotenen Weiterbildungen zu gering ist. Dabei gibt es im Fall der Lehrpersonen keinen Zusammenhang mit dem Ausmaß der bereits in Anspruch genommenen Weiterbildungen - die Anteile sind in allen der oben beschriebenen Teilgruppen annähernd identisch. Betrachtet man die Wünsche nach mehr Weiterbildungsangebot je inhaltlichem Teilbereich der digitalen Grundbildung, so ergibt sich die in Tabelle 14 dargestellte Verteilung.

\begin{tabular}{|l|c|c|}
\hline Teilbereich & $f$ & Anteil \\
\hline Ges & 23 & $41,8 \%$ \\
\hline IDM & 18 & $32,7 \%$ \\
\hline BS & 9 & $16,4 \%$ \\
\hline$M$ & 19 & $34,5 \%$ \\
\hline KSM & 13 & $23,6 \%$ \\
\hline$S$ & 14 & $25,5 \%$ \\
\hline$P$ & 7 & $12,7 \%$ \\
\hline$C T$ & 10 & $18,2 \%$ \\
\hline
\end{tabular}

Tabelle 14: Wunsch nach mehr Weiterbildungsangebot der Lehrpersonen nach inhaltlichem Teilbereich

Der höchste Bedarf an zusätzlicher Weiterbildung wird im Bereich „Gesellschaftliche Aspekte von Medienwandel und Digitalisierung“ angeführt. Am geringsten ist der Weiterbildungsbedarf in den Bereichen „Betriebssysteme und Standard-Anwendungen“, „Technische Problemlösung“ und "Computational Thinking“.

Betrachtet man den angegebenen Weiterbildungsbedarf der Lehrpersonen in Kombination mit der wahrgenommenen Wichtigkeit je Teilbereich (wie im vorgegangenen Abschnitt diskutiert), zeigt sich die in Abbildung 5 dargestellte Verteilung. Die jeweils untere Zeile je Bereich zeigt die Einschätzung der Wichtigkeit von „sehr unwichtig“ bis „sehr wichtig“ aggregiert über alle Lehrpersonen, die keinen zusätzlichen Weiterbildungsbedarf in diesem Bereich wahrnehmen. Die jeweils obere Zeile aggregiert die Wichtigkeitswahrnehmungen für alle Lehrpersonen, die zusätzlichen Weiterbildungsbedarf im jeweiligen Bereich sehen.

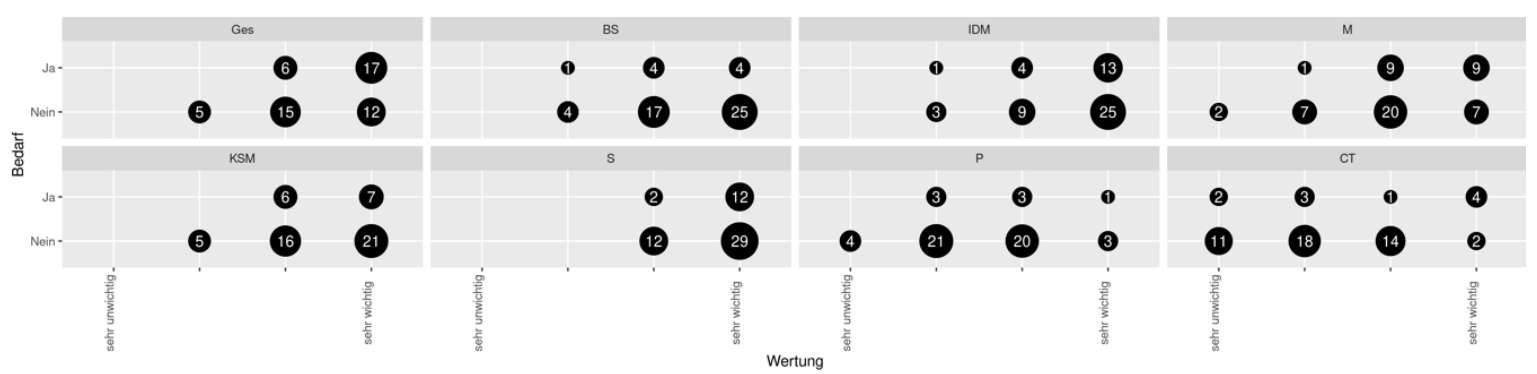

Abbildung 5: Zusätzlicher Weiterbildungsbedarf und wahrgenommene Wichtigkeit der Teilbereiche (absolute Anzahl der Nennungen)

Die wahrgenommene Wichtigkeit eines Teilbereichs scheint nur bedingt mit dem Wunsch nach verstärkten Weiterbildungsangeboten zusammenzuhängen - in allen Teilbereichen verteilen sich die Personen mit zusätzlichen Weiterbildungswünschen ähnlich über die Wichtigkeitsskala wie jene, die keinen zusätzlichen Weiterbildungsbedarf äußern. Auch existieren keine statistisch signifikanten Korrelationen zwischen der generellen Wahrnehmung des Weiterbildungsangebots bzw. dem Weiterbildungsbedarf je Teilbereich einerseits und dem Dienstalter oder dem Geschlecht der Lehrpersonen andererseits. Darüber hinaus zeigt sich ebenso hinsichtlich der geprüften Fächer der Lehrpersonen kein statistisch signifikanter Zusammenhang mit den Weiterbildungsbedarfen je Teilbereich. 


\section{Diskussion \& Schlussfolgerungen}

Das Spannungsfeld der inhaltlichen Schwerpunktsetzung bei der Vermittlung digitaler Grundbildung an Schulen, das in der Literatur wiederholt angesprochen wird (etwa in Dengel (2017) oder Brandhofer (2014)), trat auch in der Untersuchung deutlich sichtbar zu Tage. Während der Lehrplan die auch in der Literatur angeführten Themenfelder breit abdeckt, zeigt sich in der Umsetzung ein heterogeneres Bild. Die acht im Lehrplan abgebildeten Teilbereiche werden sowohl von Schulleitungen als auch von Lehrpersonen als unterschiedlich wichtig wahrgenommen. Im Sinne des Haushaltens mit den verfügbaren Zeitressourcen ist davon auszugehen, dass den unterschiedlichen Teilbereichen damit auch unterschiedlich viel Aufmerksamkeit gewidmet wird. Auffallend ist hier, dass die beiden Teilbereiche, die aus Sicht der Lernergebnisse der informatischen Perspektive (Dengel, 2017) zuzuordnen sind („Computational Thinking“, "Technische Problemlösung“), von einem überwiegenden Teil der Lehrpersonen und auch der Schulleitungen als weniger wichtig erachtet werden als jene Teilbereiche, die sich mit Medienbildung und Anwendungskompetenz beschäftigen.

Auch die Priorisierung über die Schulstufen der Sekundarstufe 1 hinweg unterstreicht dies - hier zeigt sich, dass die beiden genannten Bereiche, wenn überhaupt, dann eher in der letzten Schulstufe als relevant erachtet werden (so nennen etwa 19 der 64 teilnehmenden Lehrpersonen "Computational Thinking" als einen Bereich, der frühestens in der 8 . Schulstufe oder danach adressiert werden sollte, 7 bezeichnen ihn als generell „nicht notwendig" - dies sind insgesamt über $40 \%$ der Teilnehmer*innen). Dies läuft den Forderungen nach interdisziplinären Verankerungen der Medienbildung, der informatischen Bildung (Herzig, 2016) und der Anwendungskompetenz (Dengel, 2017) sowie der zentralen Rolle des Computational Thinking, die in der Literatur wiederholt als notwendig genannt werden (Brandhofer \& Wiesner, 2018; Steiner \& Himpsl-Gutermann, 2020), entgegen - derartige Modelle scheinen in der Praxis noch nicht verankert zu sein.

Auffällig ist auch, dass der fachliche Hintergrund der Lehrpersonen keinen Einfluss auf die Beurteilung der Wichtigkeit der Teilbereiche zu haben scheint. Auch Geschlecht und Dienstalter zeigen hier keinen Einfluss. Dies ist insofern interessant, als dass zu vermuten ist, dass unterschiedliche Fächer bei integrativen Unterrichtsformen sich unterschiedlich gut für die Vermittlung einzelner Teilbereiche eignen - im Datensatz zeigen sich hier aber keine Auffälligkeiten. Im konkreten Engagement von Lehrpersonen beim Unterrichten der "Digitalen Grundbildung" ist ein hoher Anteil von Lehrpersonen zu finden, die im Unterrichtsfach "Mathematik" geprüft sind (mehr als $50 \%$ der Teilnehmer*innen), auch im Falle von integrativem Unterricht zur digitalen Grundbildung wird Mathematik am häufigsten als jenes Fach genannt, das sich für eine diesbezügliche Umsetzung eignen würde (von 47,5\% der Teilnehmer*innen). Vor diesem Hintergrund überrascht die niedrige Wichtigkeitswahrnehmung des Teilbereichs "Computational Thinking“, dessen Lernergebnisse im Lehrplan im Bereich der Algorithmen umfassend anschlussfähig zu mathematischen Inhalten wären. Betrachtet man nur die Teilmenge der Lehrpersonen, die in Mathematik geprüft sind, ist auch dort "Computational Thinking" jener Teilbereich, der im Durchschnitt noch hinter „Technische Problemlösung“ am wenigsten wichtig eingestuft wird.

Eine Erörterung der Gründe für die geringe Wichtigkeitswahrnehmung der informatisch/technisch ausgerichteten Teilbereiche "Technische Problemlösung" und vor allem „Computational Thinking" ist auf Basis der empirischen Befunde nicht trivial. In der Zusammenschau mit den fachlichen Hintergründen der teilnehmenden Lehrpersonen, den artikulierten Weiterbildungsbedarfen sowie Formulierungen der Lernergebnisse der Teilbereiche im Lehrplan lassen sich drei Vermutungen ableiten, die in weiteren Studien gezielt untersucht werden könnten:

Zum Ersten scheinen die Benennungen der Teilbereiche und zum Teil auch die Lernergebnisse unterschiedlich konkret und unmittelbar auf reale Handlungsfelder abbildbar zu sein. So existiert der Begriff „Computational Thinking" zwar prinzipiell seit mehr als 40 Jahren (Papert, 1980), wird aber erst in den letzten Jahren im Kontext von anzustrebenden Lernergebnissen breiter rezipiert (Steiner \& Himpsl-Gutermann, 2020) und ist auch in der Fachliteratur noch von einem heterogenen Begriffsverständnis geprägt (ibid.). Eine für Entscheidungsträger (hier: Schulleitungen und Lehrpersonen) unklare begriffliche Natur eines Teilbereichs kann dazu führen, ihn zu Gunsten anderer, klarer abgrenzbarer Teilbereiche hintanzustellen.

Zum Zweiten kann die Wahrnehmung der eigenen Kompetenzen von Lehrpersonen in unterschiedlichen Teilbereichen ähnliche Effekte haben - eine weniger hohe Priorisierung von Teilbereichen, die nicht im eigenen wahrgenommenen Kompetenzbereich liegen, kann ebenfalls zu dem beobachteten Ungleichgewicht zu Ungunsten der informatisch-technischen Aspekte in der Umsetzung der verbindlichen Übung „Digitale Grundbildung" führen.

Zum Dritten ist anzunehmen, dass ob der Breite der abzudeckenden Themen auch eine Priorisierung nach wahrgenommener Relevanz der Teilbereiche für die Lebensrealitäten der Schüler*innen erfolgen muss (Reisinger, 2018). Dafür spricht etwa die hohe Wichtigkeitswahrnehmung und Verankerung des Teilbereichs 
"Sicherheit", der - betrachtet man die inkludierten Lernergebnisse - unmittelbar die alltägliche Medien- und Gerätenutzung der Schüler*innen beeinflussen kann und damit anschlussfähig zu deren Lebensrealität ist (Steiner \& Himpsl-Gutermann, 2020).

Bei der Interpretation der Ergebnisse dieser Studie sind einige Einschränkungen zu beachten. Zum Ersten war die Erhebung auf das Bundesland Vorarlberg beschränkt. Obwohl in den erhobenen potentiellen Einflussfaktoren keine Auffälligkeiten zu erkennen sind, bleibt dennoch offen, inwieweit die Erkenntnisse auf die gesamtösterreichische Situation umlegbar sind. Zum Zweiten ist zu beachten, dass die Untersuchung vor den durch COVID-19 bedingten Einstellungen des Präsenzbetriebs stattgefunden hat. Insofern wäre interessant, ob insbesondere die Wichtigkeitswahrnehmungen, aber auch der Bedarf an zusätzlichen Weiterbildungen sich nach dieser Phase verändert haben - dies sollte in weiteren Untersuchungen adressiert werden.

Trotz aller Einschränkungen identifiziert die vorliegende Studie dennoch einige Auffälligkeiten, die einerseits zu weiterem Forschungsbedarf führen, andererseits aber auch Implikationen für die Schulpraxis haben. So ist die geringe Wichtigkeitswahrnehmung und Priorisierung bei der konkreten Umsetzung der Themenbereiche, die sich mit der informatischen Perspektive digitaler Kompetenzen (Dengel, 2017) im Allgemeinen und Computational Thinking (bei aller inhaltlicher Heterogenität, siehe dazu etwa Wiesner \& Schreiner (2020); Dengel \& Heuer, (2018)) im Speziellen beschäftigen, auffällig. Über Gründe dafür können auf Basis dieser Studie nur Vermutungen angestellt werden - eine detailliertere Exploration derselben ist - im Sinne der Ermöglichung eines integrierten, multi-perspektivischen Unterrichts (Brandhofer \& Wiesner, 2018) - ein relevantes Forschungsdesiderat. Für die unmittelbare Schulpraxis macht die Studie ein bislang - bewusst oder unbewusst - in Kauf genommenes Ungleichgewicht in der Umsetzung der verbindlichen Übung „Digitale Grundbildung“ sichtbar, das bei der Planung zukünftiger Implementierungen derselben berücksichtigt werden sollte. Für künftige Überarbeitungen von einschlägigen Lehrplänen geben die Studienergebnisse Hinweise darauf, dass die aktuell abgebildete thematische Breite in Kombination mit der flexiblen Ausgestaltbarkeit der Umsetzungsformen nur bedingt dazu geeignet ist, eine kohärente und gleichmäßige Kompetenzbildung in allen Teilbereichen sicherzustellen.

\section{Literatur}

Amt der Vorarlberger Landesregierung (2019). Schulstatistik 2017/2018. Online unter https://vorarlberg.at/documents/302033/472229/Schulstatistik+2017 18+.pdf/7fe1563a-01f1-9a43-

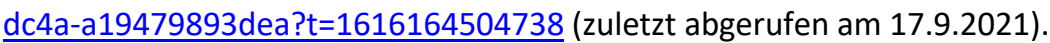

Barberi, A., Swertz, C., \& Zuliani, B. (2018). "Schule 4.0” und medialer Habitus. Medienimpulse, 56(2). https://doi.org/10.21243/mi-02-18-06

BMBWF (2018). Verordnung des Bundesministers für Bildung, Wissenschaft und Forschung, mit der die Verordnung über die Lehrpläne der Neuen Mittelschulen sowie die Verordnung über die Lehrpäne der allgemeinbildenden höheren Schulen geändert werden (BGBI. II Nr. 71/2018, Teil II). Online unter https://www.ris.bka.gv.at/eli/bgbl/ll/2018/71/20180419 (zuletzt abgerufen am 17.9.2021).

Brandhofer, G. (2014). Ein Gegenstand „Digitale Medienbildung und Informatik“ - notwendige Bedingung für digitale Kompetenz? R\&E-SOURCE, 1, 109-119.

Brandhofer, G., \& Wiesner, C. (2018). Medienbildung im Kontext der Digitalisierung: Ein integratives Modell für digitale Kompetenzen. R\&E-SOURCE, 10.

Brandhofer, G., Baumgartner, P., Ebner, M., Köberer, N., Trültzsch-Wijnen, C., \& Wiesner, C. (2019). Bildung im Zeitalter der Digitalisierung. In S. Breit, F. Eder, K. Krainer, C. Schreiner, A. Seel, \& C. Spiel (Eds.), Nationaler Bildungsbericht Österreich 2018. Fokussierte Analysen bildungspolitischer Schwerpunktthemen (Vol. 2, pp. 307-362). Leykam.

Dengel, A. (2017). Digitale Bildung: ein interdisziplinäres Verständnis zwischen Medienpädagogik und Informatik. MedienPädagogik: Zeitschrift Für Theorie Und Praxis Der Medienbildung, 33, 11-26. https://doi.org/10.21240/mpaed/33/2018.10.30.x

Dengel, A., \& Heuer, U. (2018). A curriculum of computational thinking as a central idea of information \& media literacy. Proceedings of the 13th Workshop in Primary and Secondary Computing Education, 20. https://doi.org/10.1145/3265757.3265777

Ferrari, A. (2013). DIGCOMP: A framework for developing and understanding digital competence in Europe (JRC Scientific and Policy Reports). Publications Office of the European Union Luxembourg.

Gesellschaft für Informatik e.V. (Hrsg.) (2016). Dagstuhl-Erklärung. Bildung in der digital vernetzten Welt. Online unter https://gi.de/fileadmin/GI/Hauptseite/Themen/Dagstuhl-Erkla_rung_2016-03-23.pdf (zuletzt abgerufen am 17.9.2021). 
Herzig, B. (2016). Medienbildung und Informatische Bildung - Interdisziplinäre Spurensuche. MedienPädagogik: Zeitschrift Für Theorie Und Praxis Der Medienbildung, 25(0), 59-79. https://doi.org/10.21240/mpaed/25/2016.10.28.x

Papert, S. A. (1980). Mindstorms: Children, computers, and powerful ideas. Basic books.

Reisinger, M. (2018). Digitalisierungsstrategie Schule 4.0. Neue Entwicklungen in der Vermittlung digitaler Kompetenzen an Österreichs Schulen. In Antonia Darilion, Martina Gaisch \& Schutti-Pfeil Gisela (Hrsg.), 6. Tag der Lehre der FH OÖ 2018 (S. 48-51).

Schelhowe, H. (2007). Technologie, Imagination und Lernen. Waxmann Verlag.

Steiner, M., \& Himpsl-Gutermann, K. (2020). Computational Thinking und Kontextorientierung. Medienimpulse, 58(1). https://doi.org/10.21243/mi-01-20-21

Swertz, C. (2018). Digitale Grundbildung im Pilotversuch - Beobachtungen einer entstehenden Praxis. Medienimpulse, 56(3). https://doi.org/10.21243/mi-03-18-11

Tulodziecki, G. (2016). Konkurrenz oder Kooperation? Zur Entwicklung des Verhältnisses von Medienbildung und informatischer Bildung. MedienPädagogik: Zeitschrift Für Theorie Und Praxis Der Medienbildung, 25(0), 7-25. https://doi.org/10.21240/mpaed/25/2016.10.25.x

Wiesner, C., \& Schreiner, C. (2020). Digitale Kompetenzen: Computational Thinking als Basis eines Kompetenzmodells. In Bildung und Digitalisierung (pp. 29-50). https://doi.org/10.5771/9783748906247-29

Wing, J. M. (2006). Computational thinking. Communications of the ACM, 49(3), 33-35. https://doi.org/10.1145/1118178.1118215 